\title{
Association of JMJD2B and Hypoxia-Inducible Factor 1 Expressions with Poor Prognosis in Osteosarcoma
}

\author{
Xujian Liu, ${ }^{1}$ Qianqian Zhang, ${ }^{2}$ Yi Zhao, ${ }^{1}$ Jianjun Xun, ${ }^{1}$ Hongzeng Wu, ${ }^{1}$ and Helin Feng $\mathbb{D}^{1}$ \\ ${ }^{1}$ Department of Orthopedics, The Fourth Hospital of Hebei Medical University, 12 Health Road, Shijiazhuang, Hebei 050011, China \\ ${ }^{2}$ Department of Gynecology, Hebei Medical University Second Affiliated Hospital, 215 Heping Road, Shijiazhuang, \\ Hebei 050011, China
}

Correspondence should be addressed to Helin Feng; helinfeng0311@163.com

Received 24 November 2019; Accepted 25 June 2020; Published 31 July 2020

Academic Editor: Consuelo Amantini

Copyright @ 2020 Xujian Liu et al. This is an open access article distributed under the Creative Commons Attribution License, which permits unrestricted use, distribution, and reproduction in any medium, provided the original work is properly cited.

Background. JMJD2B has been reported to be implicated in malignant tumors. This study is aimed at exploring the expression and prognostic significance of JMJD2B in osteosarcoma and its association with hypoxia-inducible factor 1 (HIF1). Methods. The histopathological and clinical characteristics were retrospectively reviewed from 53 osteosarcoma patients. JMJD2B and HIF1 were examined by immunohistochemical staining of paraffin-embedded osteosarcoma samples, and their association with clinical characteristics was examined by Spearman's test. Overall survival was examined by Kaplan-Meier analysis, and prognostic factors were identified by univariate and multivariate regression analyses. Results. JMJD2B and HIF1 expression levels were both significantly associated with Enneking stage, distant metastasis, and neoadjuvant chemotherapy, and the JMJD2B and HIF1 expressions were positively correlated $(p<0.001, R=0.752)$. In addition, univariate analysis showed that the expression of both JMJD2B and HIF1 was significantly associated with overall survival, but multivariate analysis showed that only JMJD2B expression was significantly associated with overall survival in osteosarcoma patients. Conclusions. JMJD2B and HIF1 expression levels show significant correlation with osteosarcoma progression, and JMJD2B could predict poor prognosis of osteosarcoma patients.

\section{Introduction}

Osteosarcoma (OS) is a common malignant bone tumor [1]. Despite curative resection of primary tumor, around $40 \%$ of OS patients develop isolated pulmonary metastases during the clinical course of OS [1]. There are limited novel therapeutic options, and the survival rate of patients with OS has not improved in the past decades. Better prognostic factors are important to improve the survival of patients with refractory OS.

Hypoxia-inducible factor 1 (HIF1) plays an important role in the response to low oxygen of cancer cells [2-4]. HIF1 help cancer cells adapt to hypoxia and promotes tumorigenesis [5]. HIF1 is overexpressed in many solid tumors, including OS, leading to unfavorable clinical outcome and poor survival [5]. Jumonji domain-containing pro- tein 2B (JMJD2B) is a member of the JMJD2 family of histone demethylases and is directly regulated by HIF1 [6]. JMJD2B contains the catalytic JmjC domain to demethylate tri- and di-methylated lysine 9 (H3K9me3/2) on histone $\mathrm{H} 3$ [7]. JMJD2B is overexpressed in a variety of cancers including the liver, colon, lung, and gastric cancer as well as acute myeloid leukemia [8-11]. However, the association of the HIF1 and JMJD2B expressions and their significance in OS patients remain unclear. In this study, we aimed to explore the expression and prognostic significance of JMJD2B and HIF1 in OS.

\section{Materials and Methods}

2.1. Subjects. This study was approved by the ethics committee of the Fourth Hospital of Hebei Medical University, and 
TABLE 1: Clinicopathological variables and HIF1 and JMJD2B expressions in OS patients.

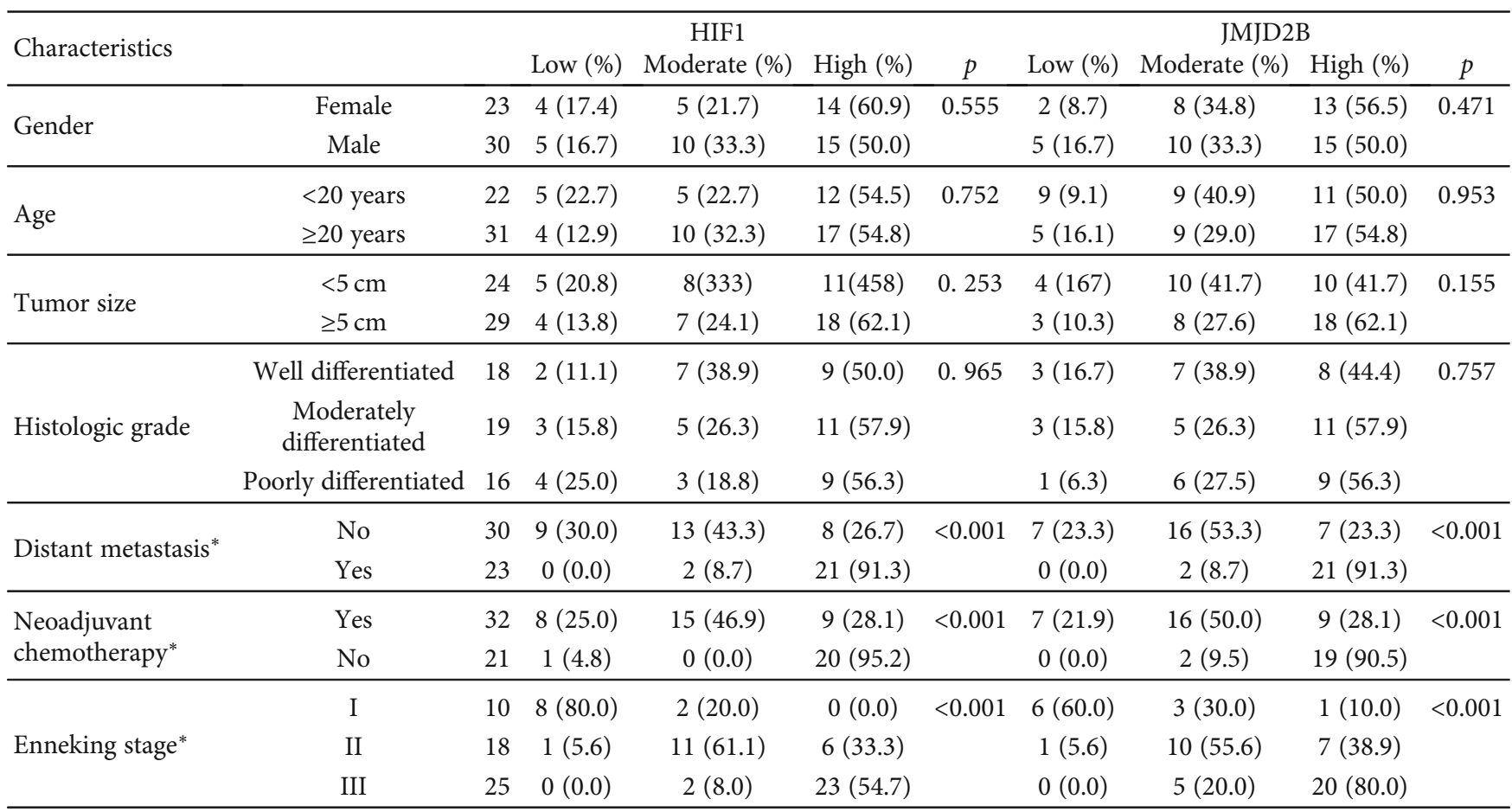

The Spearman-rho test was used. ${ }^{*} p<0.05$.

all subjects signed a written informed consent. OS samples were collected from 53 patients who visited the Fourth Hospital of Hebei Medical University between 2005 and 2013. Their histopathological and clinical characteristics were collected from medical records. No patients received radiotherapy or chemotherapy before surgery. The patients were grouped based on the age, gender, tumor size $(<5 \mathrm{~cm}$ and $\geq 5 \mathrm{~cm}$ ), histological grade, Enneking stage (I-III), and neoadjuvant chemotherapy status.

2.2. Immunohistochemistry Analysis. Immunohistochemical staining was performed on OS tissues using HIF1 antibodies ( $1: 100$; Abcam, USA) and JMJD2B antibodies (1:100; Abcam, USA) following the protocols described previously [12]. The number of positively stained cells in five randomly selected fields was analyzed by three pathologists blindly. The staining intensity was evaluated as follows: 0 , no staining; 1 , beige; 2 , darker beige; and 3, tan. The staining extent was scored as follows: $0,0 \%$ stained; $1,1 \%$ to $25 \%$ stained; $2,26 \%$ to $50 \%$ stained; and 3, $51 \%$ to $100 \%$ stained. The final scores were calculated by multiplying intensity score and extent score and judged as follows: 0 to 2 , low staining $(-/+) ; 3$ to 5 , medium staining $(++)$; and 6 to 9 , high staining $(+++)$.

2.3. Statistical Analysis. Potential prognostic factors were analyzed by $\chi^{2}$ test. Correlations of histological and clinical variables were analyzed by Spearman's rho test. Univariate and multivariate analyses were conducted using Cox proportional hazards regression analysis. Survival curves were plotted by the Kaplan-Meier method. All statistical analyses were conducted using SPSS 25.0 software, and significance was set at $p<0.05$.

\section{Results}

3.1. Characteristics of OS Patients. The characteristics of OS patient were summarized in Table 1, their mean age was 35 years (range, 9-75 years), and median overall survival was 21 months (range, 2-91 months).

3.2. Correlation of HIF1 and JMJD2B Expressions with Clinicopathological Characteristics of OS Patients. Correlation of the HIF1 and JMJD2B expressions with clinicopathological characteristics of OS patients was presented in Table 1. Representative images of HIF1 and JMJD2B staining were shown in Figure 1. Both HIF1 and JMJD2B staining were primarily in the nucleus, but the staining scores varied from low to high among OS tissues. Specifically, the HIF1 expression was low in $17.0 \%$ (9/53), moderate in $28.3 \%$ $(15 / 53)$, and high in $54.7 \%(29 / 53)$ of OS tissues, while the JMJD2B expression was low in $13.2 \%$ (7/53), moderate in $34.0 \%(18 / 53)$, and high in $52.8 \%(28 / 53)$ of OS tissues. Both HIF1 and JMJD2B showed significant association with distant metastasis $(p<0.001)$, Enneking stage $(p<0.001)$, and treatment with neoadjuvant chemotherapy $(p<0.001)$ but showed no significant association with other clinicopathological parameters. Notably, we observed a significant positive correlation between the HIF1 and JMJD2B expressions $(p<0.001)$ (Table 2).

3.3. Correlation of HIF1 and JMJD2B Expressions with Poor Overall Survival of OS Patients. Table 3 showed the results 


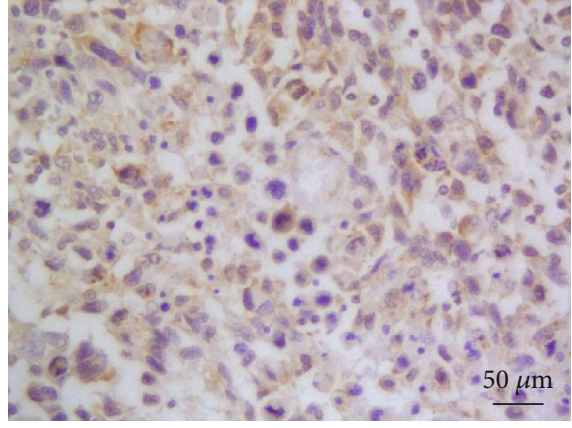

(a)

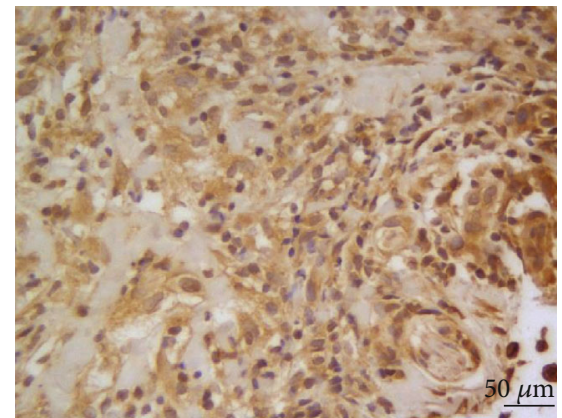

(c)

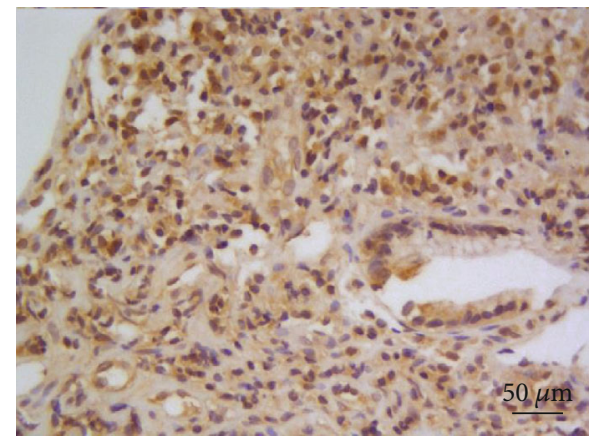

(e)

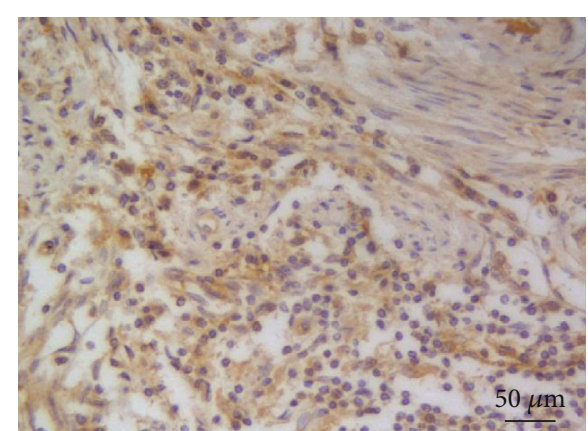

(b)

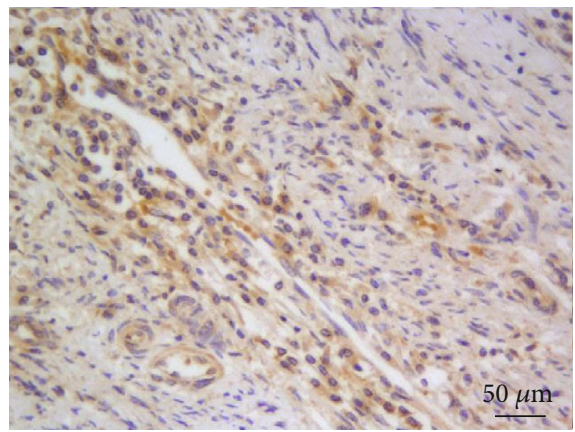

(d)

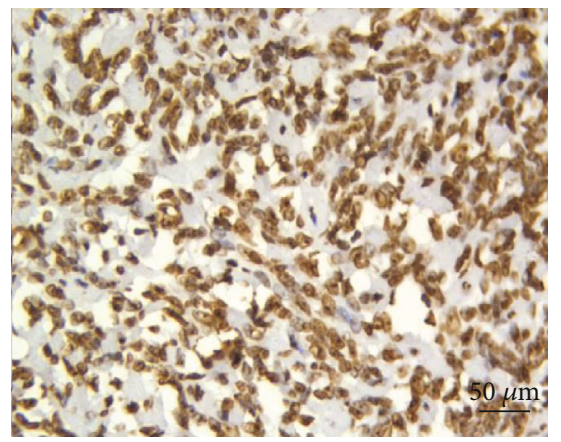

(f)

FIGURE 1: Representative immunohistochemical staining of HIF1 and JMJD2B. (a) Low expression of HIF1 in OS. (b) Moderate expression of HIF1 in OS. (c) High expression of HIF1 in OS. (d) Low expression of JMJD2B in OS. (e) Moderate expression of JMJD2B in OS. (f) High expression of JMJD2B in OS. Cells with positive expression were stained brown.

TABLE 2: Correlation of HIF1 and JMJD2B expressions.

\begin{tabular}{|c|c|c|c|c|c|}
\hline \multirow{2}{*}{ Characteristics } & & \multicolumn{3}{|c|}{ JMJD2B } & \multirow{2}{*}{$p$} \\
\hline & & Low (\%) & Moderate (\%) & High (\%) & \\
\hline \multirow{3}{*}{ HIFI } & Low & $5(55.6)$ & $3(33.3)$ & $1(11.1)$ & $<0.001$ \\
\hline & Moderate & $2(13.3)$ & $11(73.3)$ & $2(13.3)$ & \\
\hline & High & $0(0.0)$ & $4(13.8)$ & $25(86.2)$ & \\
\hline
\end{tabular}

The Spearman-rho test was used. ${ }^{*} p<0.05$.

of univariate regression analysis of overall survival. The age, gender, tumor size, and histological grade were not significant predictors of overall survival, but distant metastasis $(p<0.001)$, treatment with neoadjuvant chemotherapy $(p<0.001)$, and higher Enneking stage $(p<0.001)$ predicted shorter overall survival (Figure 2). Furthermore, higher HIF1 $(p<0.001)$ and JMJD2B $(p=0.001)$ expressions showed significant association with shorter overall survival (Table 3, Figure 2).

3.4. Higher JMJD2B Expression Predicted Poor Survival of OS Patients. Table 4 showed the results of multivariate 
TABLE 3: Univariate Cox proportional regression analysis for the association of clinicopathological factors with overall survival of OS patients.

\begin{tabular}{|c|c|c|c|c|c|}
\hline Characteristics & & & $\mathrm{HR}$ & $\begin{array}{c}\text { OS } \\
95 \% \mathrm{CI}\end{array}$ & $p$ \\
\hline \multirow{2}{*}{ Gender } & Female & 23 & 1 & & 0.214 \\
\hline & Male & 30 & 0.677 & $0.366-1.252$ & \\
\hline \multirow{2}{*}{ Age } & $<20$ years & 22 & 1 & & 0.865 \\
\hline & $\geq 20$ years & 31 & 0.948 & $0.511-1.759$ & \\
\hline \multirow{2}{*}{ Tumor size } & $<5 \mathrm{~cm}$ & 24 & 1 & & 0.492 \\
\hline & $\geq 5 \mathrm{~cm}$ & 29 & 1.240 & $0.671-2.291$ & \\
\hline \multirow{3}{*}{ Histologic grade } & Well differentiated & 18 & 1 & & 0.988 \\
\hline & Moderately differentiated & 19 & 0.998 & $0.478-2.080$ & \\
\hline & Poorly differentiated & 16 & 1.053 & 0.499-1.053 & \\
\hline \multirow{2}{*}{ Distant metastasis* } & No & 30 & 1 & & $<0.001$ \\
\hline & Yes & 23 & 5.311 & $2.403-11.736$ & \\
\hline \multirow{2}{*}{ Neoadjuvant chemotherapy* } & Yes & 32 & 1 & & 0.002 \\
\hline & No & 21 & 3.118 & $1.522-6.389$ & \\
\hline \multirow{3}{*}{ Enneking stage* } & $\mathrm{I}$ & 10 & 1 & & $<0.001$ \\
\hline & II & 9 & 12.605 & $2.643-60.109$ & \\
\hline & III & 25 & 51.909 & $9.949-270.831$ & \\
\hline \multirow{3}{*}{ HIF1 } & Low & 9 & 1 & & $<0.001$ \\
\hline & Moderate & 15 & 10.589 & $2.230-50.275$ & \\
\hline & High & 29 & 44.344 & $8.280-237.493$ & \\
\hline \multirow{3}{*}{ JMJD2B } & Low & 7 & 1 & & $<0.001$ \\
\hline & Moderate & 18 & 20.018 & $2.470-162.224$ & \\
\hline & High & 28 & 110.895 & 11.857-1037.165 & \\
\hline
\end{tabular}

OS: overall survival; HR: hazard ratio; 95\% CI: 95\% confidence interval. ${ }^{*} p<0.05$.

regression analysis of factors associated with overall survival revealed by univariate analysis. For multivariate analysis, only the JMJD2B expression $(p<0.001)$, neoadjuvant chemotherapy $(p<0.001)$, and Enneking stage $(p<0.001)$ were identified as significant prognostic factors in OS patients. OS patients with high Enneking stage, history of neoadjuvant chemotherapy, and high JMJD2B expression had significantly increased risk of mortality (log-rank $p<0.001$; Figure 2).

\section{Discussion}

To our knowledge, this is the first study to explore the expression pattern and prognostic significance of HIF1 and JMJD2B in OS patients. We found that higher HIF1 and JMJD2B levels showed a significant correlation with unfavorable clinical variables of OS patients. In addition, the HIF1 and JMJD2B expressions were positively correlated, and both HIF1 and JMJD2B were positively correlated with Enneking stage and distant metastasis. Moreover, univariate analysis showed a significant association of high HIF1 and JMJD2B expression levels with shorter overall survival in OS patients, and the JMJD2B expression was an independent prognostic indicator of
OS patients. Overall, these results suggest that JMJD2B is a significant prognostic factor of OS.

HIF1 is overexpressed in a variety of solid tumors [13]. Moreover, our previous study indicated that HIF1 was overexpressed and possibly mediated hypoxia-induced autophagic activation in human OS tissues [14]. In this study, we confirmed that HIF1 was highly expressed in OS tissues and associated with poor survival, in agreement with previous findings that high HIF1 expression indicated poor outcomes in several types of cancer $[15,16]$.

JMJD2B demethylates methylation marks on $\mathrm{H} 3 \mathrm{~K} 9$ and $\mathrm{H} 3 \mathrm{~K} 36$, resulting in the activation of transcription [17]. HIF1 can regulate the transcription of JMJD2B due to the presence of hypoxia response elements in its promoter region [6]. JMJD2B also regulates cell proliferation and promotes bladder and lung cancer cell growth by modulating cyclin-dependent kinase 6 [18-20]. In addition, a previous study showed that significant upregulation of JMJD2B in tumor tissues promoted the expression of fibroblast growth factor 2 and became a risk factor for the development of OS [11]. In this study, we confirmed that JMJD2B could be a new prognostic marker of OS. However, the mechanisms underlying the regulation of HIF1 and JMJD2B in OS remain to be elucidated. 


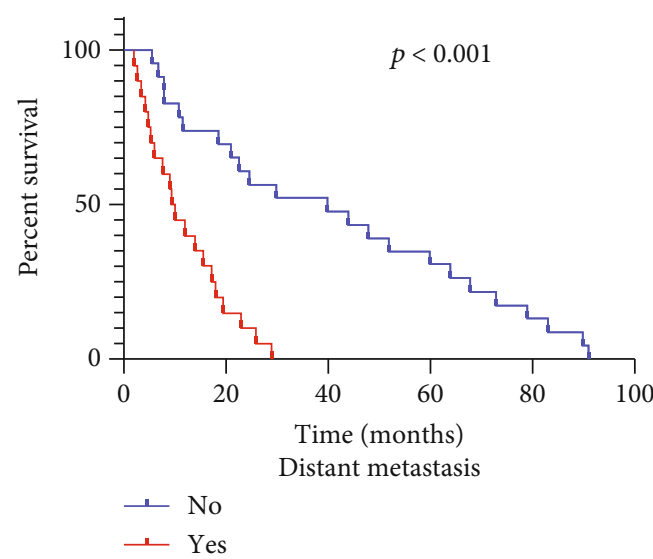

(a)

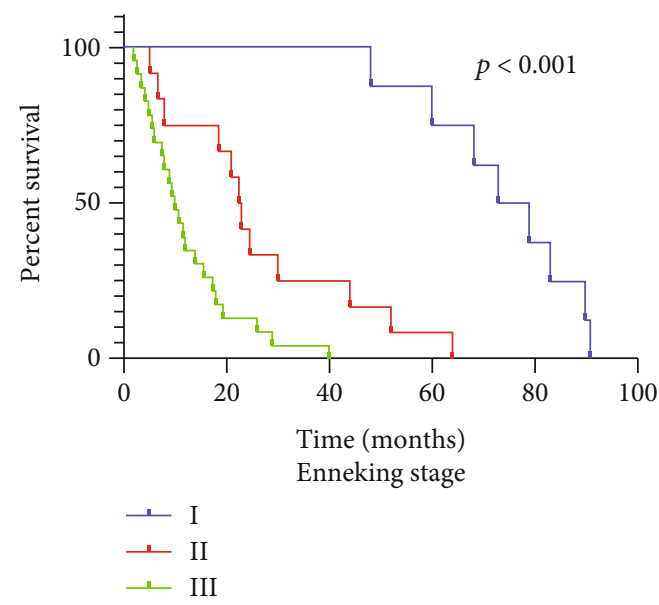

(c)

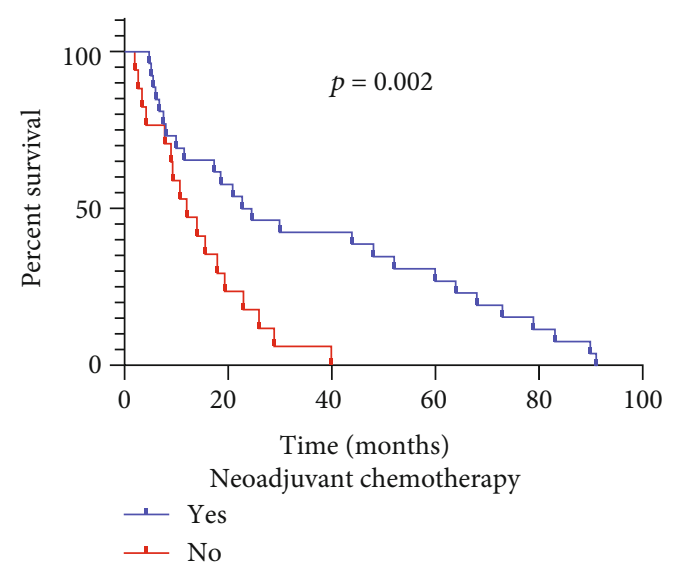

(b)

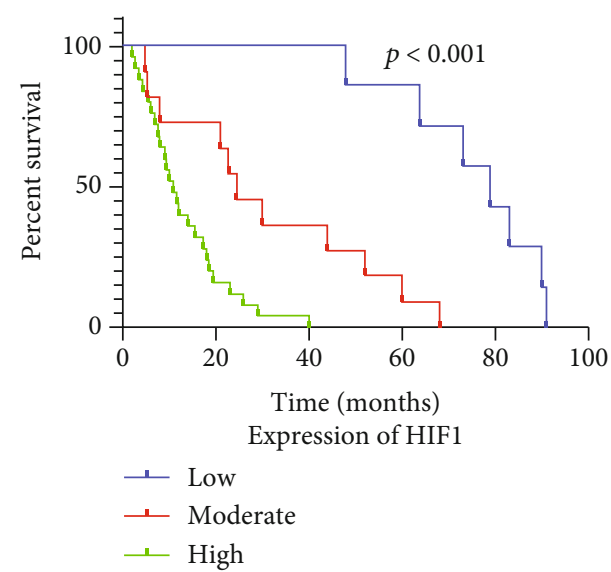

(d)

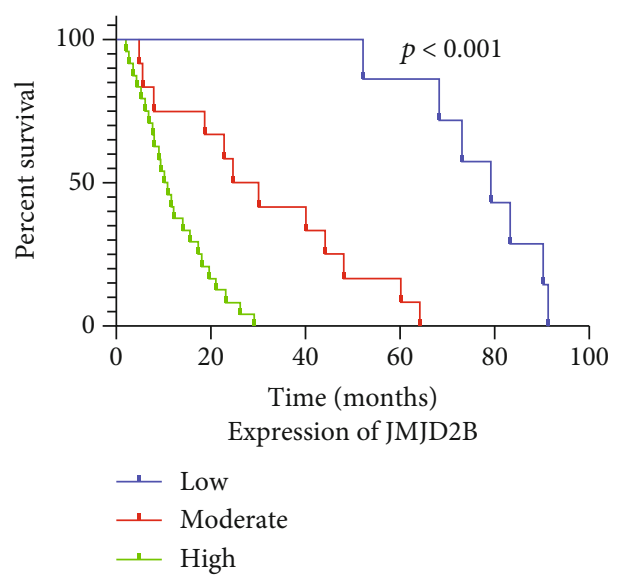

(e)

Figure 2: Overall survival curves for OS patients. Association of overall survival with (a) distant metastasis, (b) neoadjuvant chemotherapy, (c) Enneking stage, (d) HIF1 expression, and (e) JMJD2B expression. $p$ values were determined by comparing survival distributions using the log-rank test.

The present study has several limitations. First, immunohistochemical analysis is semiquantitative and needs further validation by real-time PCR. Second, our sample size was small, and our conclusion should be validated in larger samples.
In conclusion, HIF1 and JMJD2B are highly expressed in OS tissues and associated with unfavorable clinical characteristics and poor prognosis of OS patients. JMJD2B could a new clinical prognostic factor for OS patients. 
TABle 4: Multivariate Cox proportional regression analysis for the association of clinicopathological factors with overall survival of OS patients.

\begin{tabular}{lccc}
\hline Characteristics & HR & OS & CI \\
\hline Neoadjuvant chemotherapy* $^{*}$ & 0.424 & $0.185-0.972$ & 0.043 \\
Enneking stage* $^{*}$ & 4.069 & $1.685-9.826$ & 0.002 \\
JMJD2B* & 4.002 & $1.492-10.735$ & 0.006
\end{tabular}

OS: overall survival; HR: hazard ratio; $95 \%$ CI: $95 \%$ confidence interval. ${ }^{*} p$ $<0.05$.

\section{Data Availability}

All data and material are available upon request.

\section{Conflicts of Interest}

The authors declare no conflicts of interest.

\section{Authors' Contributions}

HF designed the study. XL, QZ, and YZ collected the samples and performed the analysis. JX and HW performed the statistical analysis. All authors wrote and approved the final manuscript. Xujian Liu and Qianqian Zhang contributed equally to this work.

\section{Acknowledgments}

This work was supported by the Key Project of MedicalScience Research of Hebei Province (20180564) and the Project of the Natural Science Foundation of Hebei Province (H2019206309).

\section{References}

[1] M. A. Anwar, C. El-Baba, M. H. Elnaggar et al., "Novel therapeutic strategies for spinal osteosarcomas," Seminars in Cancer Biology, vol. 64, pp. 83-92, 2020.

[2] H. Li, C. Liao, W. Weng, H. Zhong, and T. Zhou, "Association of hypoxia-inducible factor- $1 \alpha$ (HIF $1 \alpha) 1772 \mathrm{C} / \mathrm{T}$ gene polymorphism with susceptibility to renal cell carcinoma/prostate cancer," Biocell, vol. 44, no. 2, pp. 257-262, 2020.

[3] D. S. Kahraman, G. Diniz, S. Sayhan et al., "The clinicopathologic significance of hypoxia inducible factor 1 alpha expression in ovarian serous tumors," European Journal of Gynaecological Oncology, vol. 40, pp. 242-245, 2019.

[4] H. Zhao, Y. Wu, Y. Chen, and H. Liu, "Clinical significance of hypoxia-inducible factor 1 and VEGF-A in osteosarcoma," International Journal of Clinical Oncology, vol. 20, no. 6, pp. 1233-1243, 2015.

[5] N. T. Moldogazieva, I. M. Mokhosoev, and A. A. Terentiev, "Metabolic heterogeneity of cancer cells: an interplay between HIF-1, GLUTs, and AMPK," Cancers (Basel), vol. 12, no. 4, p. $862,2020$.

[6] P.. J. Pollard, C. Loenarz, D. . R. Mole et al., "Regulation of Jumonji-domain-containing histone demethylases by hypoxia-inducible factor (HIF)-1alpha," The Biochemical Journal, vol. 416, no. 3, pp. 387-394, 2008.
[7] L. Castellini, E. J. Moon, O. V. Razorenova, A. J. Krieg, R. von Eyben, and A. J. Giaccia, "KDM4B/JMJD2B is a p53 target gene that modulates the amplitude of p53 response after DNA damage," Nucleic Acids Research, vol. 45, no. 7, pp. 3674-3692, 2017.

[8] K. Agger, S. Miyagi, M. T. Pedersen, S. M. Kooistra, J. V. Johansen, and K. Helin, "Jmjd2/Kdm4 demethylases are required for expression of Il3ra and survival of acute myeloid leukemia cells," Genes \& Development, vol. 30, no. 11, pp. 1278-1288, 2016.

[9] L. N. Fu, Y. Q. Wang, J. Tan et al., "Role of JMJD2B in colon cancer cell survival under glucose-deprived conditions and the underlying mechanisms," Oncogene, vol. 37, no. 3, pp. 389-402, 2018.

[10] J. Zhang, J. Ren, S. Hao et al., "MiRNA-491-5p inhibits cell proliferation, invasion and migration via targeting JMJD2B and serves as a potential biomarker in gastric cancer," American Journal of Translational Research, vol. 10, no. 2, pp. 525534, 2018.

[11] X. Li and S. Dong, "Histone demethylase JMJD2B and JMJD2C induce fibroblast growth factor 2: mediated tumorigenesis of osteosarcoma," Medical Oncology, vol. 32, no. 3, 2015.

[12] C. Yang and Y. Tian, "SPAG9 promotes prostate cancer growth and metastasis," Biocell, vol. 43, no. 3, pp. 207-213, 2019.

[13] P. S. Macklin, A. Yamamoto, L. Browning, M. Hofer, J. Adam, and C. W. Pugh, "Recent advances in the biology of tumour hypoxia with relevance to diagnostic practice and tissuebased research," The Journal of Pathology, vol. 250, no. 5, pp. 593-611, 2020.

[14] H. Feng, J. Wang, W. Chen et al., "Hypoxia-induced autophagy as an additional mechanism in human osteosarcoma radioresistance," J Bone Oncol., vol. 5, no. 2, pp. 67-73, 2016.

[15] V. Laurent, A. Toulet, C. Attané et al., "Periprostatic adipose tissue favors prostate cancer cell invasion in an obesitydependent manner: role of oxidative stress," Molecular Cancer Research, vol. 17, no. 3, pp. 821-835, 2019.

[16] H. J. Meyer, L. Leifels, G. Hamerla, A. K. Höhn, and A. Surov, "ADC-histogram analysis in head and neck squamous cell carcinoma. Associations with different histopathological features including expression of EGFR, VEGF, HIF- $1 \alpha$, Her 2 and p53. A preliminary study," Magnetic Resonance Imaging, vol. 54, pp. 214-217, 2018.

[17] B. D. Fodor, S. Kubicek, M. Yonezawa et al., "Jmjd2b antagonizes $\mathrm{H} 3 \mathrm{~K} 9$ trimethylation at pericentric heterochromatin in mammalian cells," Genes \& Development, vol. 20, no. 12, pp. 1557-1562, 2006.

[18] J. W. Lu, Y. J. Ho, L. I. Lin et al., “JMJD2B as a potential diagnostic immunohistochemical marker for hepatocellular carcinoma: a tissue microarray-based study," Acta Histochemica, vol. 117, no. 1, pp. 14-19, 2015.

[19] L. Duan, R. E. Perez, X. Lai, L. Chen, and C. G. Maki, “The histone demethylase JMJD2B is critical for p53-mediated autophagy and survival in Nutlin-treated cancer cells," The Journal of Biological Chemistry, vol. 294, no. 23, pp. 9186-9197, 2019.

[20] L. Duan, R. E. Perez, P. D. Chastain, M. T. Mathew, D. R. Bijukumar, and C. G. Maki, "JMJD2 promotes acquired cisplatin resistance in non-small cell lung carcinoma cells," Oncogene, vol. 38, no. 28, pp. 5643-5657, 2019. 